\title{
Schrödinger equation on the Heisenberg group
}

\author{
by \\ JACEK ZiENKIEWICZ (Wrocław)
}

\begin{abstract}
Let $L$ be the full laplacian on the Heisenberg group $\mathbb{H}^{n}$ of arbitrary dimension $n$. Then for $f \in L^{2}\left(\mathbb{H}^{n}\right)$ such that $(I-L)^{s / 2} f \in L^{2}\left(\mathbb{H}^{n}\right)$ for some $s>1 / 2$ and for every $\phi \in C_{\mathrm{c}}\left(\mathbb{H}^{n}\right)$ we have

$$
\int_{\mathbb{H}^{n}}|\phi(x)| \sup _{0<t \leq 1}\left|e^{\sqrt{-1} t L} f(x)\right|^{2} d x \leq C_{\phi}\|f\|_{W^{s}}^{2} .
$$
\end{abstract}

Introduction. Let $V_{t}$ be the Schrödinger unitary group generated by a self-adjoint, positive differential operator $L$ on $\mathbb{R}^{d}$. The degree of smoothness needed for the almost everywhere convergence of $V_{t} f$ to $f$ as $t \rightarrow 0$ has been extensively studied. In general, the result of Cowling $[\mathrm{Cw}]$ says that if $\left\|(1+L)^{s / 2} f\right\|_{L^{2}}<\infty$ for some $s>1$, then

$$
\lim _{t \rightarrow 0} V_{t} f(x)=f(x) \quad \text { a.e. }
$$

This does not depend on any other properties of $L$.

For $-L$ being the Laplace operator on $\mathbb{R}^{d}, s>1 / 2$ suffices for all $d$, and for $d=2, s>1 / 2-\delta$ is also sufficient. See [B], [Mo]. In our previous paper [Z] the Laplace operator $L$ on the Heisenberg group $\mathbb{H}^{n}$ has been studied from this point of view, and we have proved that $s>3 / 4 \operatorname{implies}(*)$. In this paper we simplify the proof of the result in [Z] and decrease the needed regularity of $f$ to $f \in W^{s}, s>1 / 2$.

The author would like to express his gratitude to M. Derencz and A. Hulanicki for their help in editing the paper.

0. Preliminaries. We identify $\mathbb{R}^{2}$ with $\mathbb{C}$ and consequently $\mathbb{R}^{2 n}$ with $\mathbb{C}^{n}$. Denote by $S(\mathbf{z}, \mathbf{w})=2 \Im(\mathbf{z} \cdot \overline{\mathbf{w}})$ the standard symplectic form on $\mathbb{R}^{2 n}$.

2000 Mathematics Subject Classification: 35S10, 42B25. 
For $m=0,1,2, \ldots$ let

$$
L_{m}(x)=\sum_{k=0}^{m}\left(\begin{array}{c}
m \\
k
\end{array}\right) \frac{(-x)^{k}}{k !}
$$

be the Laguerre polynomial of degree $m$ and for $a \neq 0$ let

$$
l_{m, a}(z)=e^{-|a||z|^{2}} L_{m}\left(2|a||z|^{2}\right)
$$

be the corresponding Laguerre function.

Let $\mathbf{m}=\left(m_{1}, \ldots, m_{n}\right)$ and $\mathbf{z}=\left(z_{1}, \ldots, z_{n}\right)$. We write

$$
q_{\mathbf{m}, a}(\mathbf{z})=l_{m_{1}, a}\left(z_{1}\right) l_{m_{2}, a}\left(z_{2}\right) \ldots l_{m_{n}, a}\left(z_{n}\right) .
$$

It is well known that the $q_{\mathbf{m}, 1}$ form an orthonormal basis of the space of polyradial functions on $\mathbb{C}^{n}$.

We denote by $d \mathbf{z}$ the Lebesgue measure on $\mathbb{C}^{n}$ and for $a \neq 0$ we define the twisted convolution

$$
f \times_{a} g(\mathbf{z})=\int f(\mathbf{z}-\mathbf{w}) g(\mathbf{w}) e^{i a S(\mathbf{z}, \mathbf{w})} d \mathbf{w}, \quad f, g \in C_{\mathbf{c}}^{\infty}\left(\mathbb{C}^{n}\right) .
$$

We have the following orthogonality relation for the Laguerre functions (cf. $[\mathrm{M}])$ :

$$
|a|^{n} q_{\mathbf{k}, a} \times_{a} q_{\mathbf{m}, a}(\mathbf{z})=\delta_{\mathbf{k}, \mathbf{m}} q_{\mathbf{m}, a}(\mathbf{z}) .
$$

Fix a real $a \neq 0$ and let

$$
Q_{\mathbf{m}, a} f(\mathbf{z})=|a|^{n} q_{\mathbf{m}, a} \times_{a} f(\mathbf{z}) .
$$

It follows from (0.1) that for a fixed $a \neq 0$ the operators $Q_{\mathbf{m}, a}$ are mutually orthogonal projectors. Moreover $\sum_{\mathbf{m}} Q_{\mathbf{m}, a}=\mathrm{Id}$ (cf. [M]).

We introduce a separate notation for the operators $Q_{\mathbf{m}, a}$ in the case $\mathbf{m}=m \in \mathbb{N}$, i.e. $n=1$. We then write

$$
Q_{\mathbf{m}, a} f=P_{m, a} f=|a| l_{m, a} \times_{a} f .
$$

The Heisenberg group $\mathbb{H}^{n}$ is defined as $\mathbb{C}^{n} \times \mathbb{R}$, with the group product $(\mathbf{z}, s)(\mathbf{w}, t)=(\mathbf{z}+\mathbf{w}, s+t+2 \Im(\mathbf{z} \cdot \overline{\mathbf{w}}))$ where $\mathbf{z}=\left(z_{1}, \ldots, z_{n}\right), z_{j}=x_{j}+i y_{j}$. Then the Lebesgue measure on $\mathbb{C}^{n} \times \mathbb{R}$ is the Haar measure on $\mathbb{H}^{n}$.

Let

$$
X_{i}=\partial_{x_{i}}+2 y_{i} \partial_{t}, \quad Y_{i}=\partial_{y_{i}}-2 x_{i} \partial_{t} \quad \text { for } 1 \leq i \leq n, \quad T=\partial_{t},
$$

and let

$$
L=\sum_{i=1}^{n}\left(X_{i}^{2}+Y_{i}^{2}\right)+T^{2}
$$

be the elliptic laplacian on $\mathbb{H}^{n}$. The closure of $L$ on $C_{\mathrm{c}}^{\infty}\left(\mathbb{H}^{n}\right)$ is a self-adjoint operator (see [NS]). Therefore $i L$ generates a group $\left\{V_{t}\right\}_{t \in \mathbb{R}}$ of unitary operators on $L^{2}\left(\mathbb{H}^{n}\right)$. We will use the following formula for $V_{t}$, valid for $f \in S\left(\mathbb{H}^{n}\right)$ 
(cf. $[\mathrm{M}])$ :

$$
V_{t} f(\mathbf{z}, u)=\sum_{\mathbf{m}} \int_{\mathbb{R}} e^{i u a} e^{i t \lambda_{|\mathbf{m}|}(a)} Q_{\mathbf{m}, a} f^{a}(\mathbf{z}) d a,
$$

where $\lambda_{|\mathbf{m}|}(a)=(2|\mathbf{m}|+n)|a|+a^{2},|\mathbf{m}|=m_{1}+\ldots+m_{k}$ and $f^{a}$ denotes the Fourier transform with respect to the central variable.

Let $s \geq 0$. We define a scale of Sobolev spaces by putting

$$
\|f\|_{W^{s}}=\left\|(I-L)^{s / 2} f\right\|_{L^{2}} .
$$

Since $Q_{\mathbf{m}, a}$ are mutually orthogonal projectors and

$$
L f(\mathbf{z}, u)=\int_{\mathbb{R}} \sum_{\mathbf{m}} e^{i u a} \lambda_{|\mathbf{m}|}(a) Q_{\mathbf{m}, a} f^{a}(\mathbf{z}) d a
$$

the Plancherel theorem applied to the variable $u$ implies

$$
\|f\|_{W^{s}}^{2}=\sum_{\mathbf{m}} \int_{\mathbb{R}}\left(1+\lambda_{|\mathbf{m}|}(a)\right)^{s}\left\|Q_{\mathbf{m}, a} f^{a}\right\|_{L^{2}\left(\mathbb{C}^{n}\right)}^{2} d a .
$$

For a more detailed exposition of the preliminary facts we refer the reader to $[\mathrm{M}]$ and $[\mathrm{Z}]$.

1. Basic lemmas. Let $0<\alpha<1$. The fractional derivative of order $\alpha$ is defined by

$$
\partial^{\alpha} f(s)=\int_{\mathbb{R}}(f(s-t)-f(s))|t|^{-(1+\alpha)} d t .
$$

Lemma 1 (Sobolev). Let $\gamma>0$ be a Schwartz function and $1 / 2<\alpha<1$. Then

$$
\sup _{-1 \leq t \leq 1}|f(t)|^{2} \leq C_{\alpha}\left(\int_{\mathbb{R}}\left|\partial^{\alpha} f(t)\right|^{2} \gamma(t) d t+\int_{\mathbb{R}}|f(t)|^{2} \gamma(t) d t\right) .
$$

For a function $\phi$, let $M_{\phi}$ denote the operator of multiplication by $\phi$. Set $B(r)=\{z:|z| \leq r\}$.

Fix $\phi \in C_{\mathrm{c}}^{\infty}(\mathbb{C})$ with $\operatorname{supp} \phi \subset B(1)$ and $|\phi(z)| \leq 1$, and define

$$
T_{m, a} f(z)=M_{\phi} P_{m, a} f(z)=\phi(z)|a| l_{m, a} \times_{a} f(z) .
$$

Since $P_{m, a}$ is an orthogonal projector we have $\left\|T_{m, a}\right\|_{L^{2} \rightarrow L^{2}} \leq 1$. The following two lemmas have been proved in $[\mathrm{Z}]$.

Lemma 2. For $4 \leq|a| \leq m+1$ we have

$$
\left\|T_{m, a}\right\|_{L^{2} \rightarrow L^{2}}^{2} \leq C\left(\frac{|a|}{m+1}\right)^{1 / 2} .
$$

Lemma 3. For $|a| \leq 4$ we have

$$
\left\|T_{m, a}\right\|^{2} \leq C\left(\frac{|a|}{m+1}\right)^{1 / 2}
$$


For the reader's convenience we include the proofs of Lemmas 2 and 3. To do this we need a number of consequences of the classical estimates for Laguerre functions, collected below.

LEMMA 4.

$$
L_{m}(\lambda x)=\sum_{k=0}^{m}\left(\begin{array}{c}
m \\
k
\end{array}\right) L_{k}(x) \lambda^{k}(1-\lambda)^{m-k} .
$$

LEMma 5. Let $1 \leq|z| \leq(m+1)^{1 / 2}$. Then

$$
\left|l_{m, 1}(z)\right| \leq C(m+1)^{-1 / 4}|z|^{-1 / 2} .
$$

Proof. Let $0<\varepsilon \leq \varphi \leq \pi / 2-\varepsilon(m+1)^{-1 / 2}$. Then by a theorem of Szegö [Sz], for $x=(4 m+2) \cos ^{2} \varphi$, we have

$$
\begin{aligned}
e^{-x / 2} L_{m}(x)= & (-1)^{m}(\pi \sin \varphi)^{-1 / 2}(\sin ((m+1 / 2)(\sin 2 \varphi-2 \varphi)+3 \pi / 4) \\
& \left.\times(x(m+1))^{-1 / 4}+(x(m+1))^{-1 / 2} O(1)\right) .
\end{aligned}
$$

Lemma 6. Let $|z| \leq 1$. Then

$$
l_{m, 1}(z)=J_{0}\left(2^{1 / 2}|z|(m+1 / 2)^{1 / 2}\right)+O\left((m+1)^{-3 / 4}\right),
$$

where $J_{0}$ is the zero Bessel function.

Proof. Follows from an asymptotic formula for the Laguerre polynomials (cf. $[\mathrm{Sz}])$ :

$$
e^{-x / 2} L_{m}(x)=J_{0}\left((2 x(m+1 / 2))^{1 / 2}\right)+O\left((m+1)^{-3 / 4}\right) .
$$

Lemma 7. There is a constant $C$ such that for $A \geq 1$ we have

$$
\int\left|l_{m, 1}(z)\right|^{2} e^{-|z|^{2} / A^{2}} d z \leq C A(m+1)^{-1 / 2} .
$$

Proof. By Lemma 5, we obtain

$$
\begin{aligned}
& \quad \int_{1 \leq|z| \leq(m+1)^{1 / 2}}\left|l_{m, 1}(z)\right|^{2} e^{-|z|^{2} / A^{2}} d z \\
& \leq C \int \frac{1}{|z|(m+1)^{1 / 2}} e^{-|z|^{2} / A^{2}} d z \leq C A(m+1)^{-1 / 2} .
\end{aligned}
$$

Also

$$
\int_{|z| \geq(m+1)^{1 / 2}}\left|l_{m, 1}(z)\right|^{2} e^{-|z|^{2} / A^{2}} d z \leq e^{-m / A^{2}} \int\left|l_{m, 1}(z)\right|^{2} d z \leq C A(m+1)^{-1 / 2} .
$$

On the other hand, by Lemma 6 , using the estimate $\left|J_{0}(x)\right| \leq C(1+|x|)^{-1 / 2}$ for the Bessel function (see $[\mathrm{Sz}]$ ) we obtain

$$
\left|l_{m, 1}(z)\right| \leq C\left(1+|z|^{1 / 2}(m+1)^{1 / 4}\right)^{-1} .
$$

Hence

$$
\int_{|z| \leq 1}\left|l_{m, 1}(z)\right|^{2} e^{-|z|^{2} / A^{2}} d z \leq C(m+1)^{-1 / 2} .
$$


Proof of Lemma 2. Since $P_{m, a}$ is an orthogonal projector, $T_{m, a} T_{m, a}^{*}=$ $M_{\phi} P_{m, a} M_{\phi}$. Hence, the kernel $K$ of $T_{m, a} T_{m, a}^{*}$ is given by the formula

$$
K\left(z_{1}, z_{2}\right)=\phi\left(z_{1}\right)|a| l_{m, a}\left(z_{1}-z_{2}\right) e^{-i a S\left(z_{1}, z_{2}\right)} \phi\left(z_{2}\right) .
$$

We write

$$
1=e^{-\left|z_{1}-z_{2}\right|^{2}} e^{\left|z_{1}-z_{2}\right|^{2}}=e^{-\left|z_{1}-z_{2}\right|^{2}} \sum_{\alpha} c_{\alpha} z_{1}^{\alpha_{1}} \bar{z}_{1}^{\alpha_{3}} z_{2}^{\alpha_{2}} \bar{z}_{2}^{\alpha_{4}} .
$$

Thus

$$
\begin{aligned}
& K\left(z_{1}, z_{2}\right) \\
= & \sum_{\alpha} c_{\alpha} z_{1}^{\alpha_{1}} \bar{z}_{1}^{\alpha_{3}} \phi\left(z_{1}\right) e^{-\left|z_{1}-z_{2}\right|^{2}}|a| l_{m, a}\left(z_{1}-z_{2}\right) e^{-i a S\left(z_{1}, z_{2}\right)} \phi\left(z_{2}\right) z_{2}^{\alpha_{2}} \bar{z}_{2}^{\alpha_{4}} .
\end{aligned}
$$

Consequently, the operator $T_{m, a} T_{m, a}^{*}$ is the sum over $\alpha$ of operators

$$
c_{\alpha} M_{\phi} M_{z_{1}^{\alpha}}^{\alpha_{1} \bar{z}_{1}^{\alpha_{3}}} T_{K_{1}} M_{z_{2}^{\alpha_{2}} \bar{z}_{2}^{\alpha_{4}}} M_{\phi}
$$

where

$$
T_{K_{1}}=f \times_{a} K_{1}, \quad K_{1}(z)=e^{-|z|^{2}}|a| l_{m, a}(z) .
$$

Since $c_{\alpha}$ converges to zero faster than exponentially, it suffices to estimate the norm of $T_{K_{1}}$. Dilating we see that the norm of $T_{K_{1}}$ is the same as the norm of the 1-twisted convolution operator by

$$
\mathcal{K}(z)=e^{-|z|^{2}|a|^{-1}} l_{m, 1}(z) .
$$

The radial function $\mathcal{K}(z)$ has a decomposition

$$
\mathcal{K}(z)=\sum_{k=0}^{\infty} c_{k, m, a} l_{k, 1}(z),
$$

where

$$
c_{k, m, a}=\int e^{-|z|^{2}|a|^{-1}} l_{k, 1}(z) l_{m, 1}(z) d z
$$

So

$$
\mathcal{K}(z) \times_{1} f(z)=\sum_{k=0}^{\infty} c_{k, m, a} P_{m, 1} f(z) .
$$

Since $P_{m, 1}, m=0,1, \ldots$, are mutually orthogonal projectors the norm of the operator $f \mapsto \mathcal{K} \times_{1} f$ is equal to

$$
\sup _{k}\left|c_{k, m, a}\right| .
$$

By the Schwarz inequality, we obtain

$$
\left|c_{k, m, a}\right| \leq\left\|e^{-|z|^{2} / 2|a|} l_{k, 1}(z)\right\|_{L^{2}}\left\|e^{-|z|^{2} / 2|a|} l_{m, 1}(z)\right\|_{L^{2}} .
$$


Now, by Lemma 7 , if $10 k \geq m$, then

$$
\left|c_{k, m, a}\right| \leq C\left(\frac{|a|}{m+1}\right)^{1 / 4}\left(\frac{|a|}{k+1}\right)^{1 / 4} \leq C\left(\frac{|a|}{m+1}\right)^{1 / 2}
$$

It remains to estimate the coefficients $c_{k, m, a}$ for $10 k \leq m$. Observe that by the definition of $l_{m, 1}(z)$, for $\lambda=\left(1+(2|a|)^{-1}\right)^{-1},(*)$ turns into

$$
c_{k, m, a}=C \int_{0}^{\infty} e^{-\lambda^{-1} x} L_{m}(x) L_{k}(x) d x
$$

Then

$$
c_{k, m, a}=C \lambda \int_{0}^{\infty} e^{-x} L_{m}(x \lambda) L_{k}(x \lambda) d x
$$

whence, in virtue of (1.1), because the $L_{k}$ form an orthonormal basis with the weight $e^{-x}$, we obtain

$$
\begin{aligned}
c_{k, m, a}= & C \lambda \sum_{s_{1}=0}^{m} \sum_{s_{2}=0}^{k}\left(\begin{array}{c}
m \\
s_{1}
\end{array}\right)\left(\begin{array}{c}
k \\
s_{2}
\end{array}\right) \lambda^{\left(s_{1}+s_{2}\right)}(1-\lambda)^{m+k-\left(s_{1}+s_{2}\right)} \\
& \times \int_{0}^{\infty} e^{-x} L_{s_{1}}(x) L_{s_{2}}(x) d x \\
= & C \lambda \sum_{s=0}^{k}\left(\begin{array}{c}
m \\
s
\end{array}\right)\left(\begin{array}{c}
k \\
s
\end{array}\right) \lambda^{2 s}(1-\lambda)^{m+k-2 s} .
\end{aligned}
$$

Now, if $|a| \geq 4$ then $2 / 3 \leq \lambda \leq 1$ so for $10 k \leq m$ we have

$$
\begin{aligned}
\left|c_{k, m, a}\right| & \leq \sum_{s=0}^{k} 2^{m} 2^{k}(1-\lambda)^{m+k-2 k} \leq k 2^{m} 2^{k} 3^{-m-k} \\
& \leq k\left(\frac{2}{3}\right)^{m+k} \leq 2^{-\varepsilon m} \leq 2^{-\varepsilon m}|a|
\end{aligned}
$$

for some positive constant $\varepsilon$.

Proof of Lemma 3. In order to estimate the norm of $T_{m, a} T_{m, a}^{*}$ we use (1.1) and the asymptotic formula for the Laguerre functions given in Lemma 6 .

Let $|a| \leq 4$. By the Taylor series expansion for $e^{i a S\left(z_{1}, z_{2}\right)}$ we have

$$
\begin{aligned}
K\left(z_{1}, z_{2}\right) & =\sum_{\alpha} z_{1}^{\alpha_{1}} \bar{z}_{1}^{\alpha_{3}} \phi\left(z_{1}\right)|a| l_{m, a}\left(z_{1}-z_{2}\right) \phi\left(z_{2}\right) z_{2}^{\alpha_{2}} \bar{z}_{2}^{\alpha_{4}} a_{\alpha}|a|^{|\alpha| / 2} \\
& =\sum_{\alpha} a_{\alpha}|a|^{|\alpha| / 2} K_{\alpha}\left(z_{1}, z_{2}\right) .
\end{aligned}
$$

Since the $a_{\alpha}$ 's decay faster than exponentially, and the norms of the operators $M_{\phi} M_{z^{\alpha}}$ grow at most exponentially, it suffices to estimate the norm of 
the operator $K$ given by the kernel

$$
\begin{aligned}
& A\left(z_{1}, z_{2}\right)=\psi\left(z_{1}\right)|a| l_{m, a}\left(z_{1}-z_{2}\right) \psi\left(z_{2}\right), \quad \text { where } \\
& \qquad \psi \in C_{\mathrm{c}}^{\infty} \text { with } \psi(z)=1 \text { on } \operatorname{supp} \phi .
\end{aligned}
$$

Now using Lemma 6 we obtain

$$
\begin{aligned}
\psi\left(z_{1}\right)|a| l_{m, a}\left(z_{1}-z_{2}\right) \psi\left(z_{2}\right)= & C \psi\left(z_{1}\right)|a| J_{0}\left(2|a|^{1 / 2}\left|z_{1}-z_{2}\right|(2 m+1)^{1 / 2}\right) \psi\left(z_{2}\right) \\
& +\psi\left(z_{1}\right) \psi\left(z_{2}\right) O\left(|a|(m+1)^{-3 / 4}\right) .
\end{aligned}
$$

Observe that the error term in the last formula gives an operator with norm of order $|a|(m+1)^{-3 / 4}$, so it is negligible.

Hence, for a function $\widetilde{\phi} \in S(\mathbb{C})$ with $\widetilde{\phi}=1$ on $\operatorname{supp} \psi-\operatorname{supp} \psi$ we write

$$
\begin{aligned}
\psi\left(z_{1}\right)|a| J_{0}\left(|a|^{1 / 2}\left|z_{1}-z_{2}\right|(2 m+1)^{1 / 2}\right) \psi\left(z_{2}\right) \\
\quad=\widetilde{\phi}\left(z_{1}-z_{2}\right) \psi\left(z_{1}\right)|a| J_{0}\left(|a|^{1 / 2}\left|z_{1}-z_{2}\right|(2 m+1)^{1 / 2}\right) \psi\left(z_{2}\right) .
\end{aligned}
$$

Thus we may drop $\psi\left(z_{1}\right), \psi\left(z_{2}\right)$ and we estimate the norm of the convolution operator by the function

$$
R=\widetilde{\phi}(z)|a| J_{0}\left(|a|^{1 / 2}|z|(2 m+1)^{1 / 2}\right) .
$$

By definition, $J_{0}$ is the Fourier transform of the normalized Lebesgue measure supported on the unit circle. Hence

$$
\widehat{R}=\widehat{\widetilde{\phi}} *|a| \mu,
$$

where $\mu$ is the normalized Lebesgue measure supported by the circle of radius $|a(2 m+1)|^{1 / 2}$. We write (using a smooth resolution of identity $1=$ $\sum_{j \in \mathbb{Z}^{2}} k(z-j)$ with supp $\left.k \subset B(2)\right)$

$$
\widehat{\widetilde{\phi}}=\sum_{j} \alpha_{j} \phi_{j}
$$

where $\sum_{j}\left|\alpha_{j}\right|<\infty,\left\|\phi_{j}\right\|_{L^{\infty}} \leq 1$ and the support of $\phi_{j}$ is contained in the disc of radius two. A trivial geometric argument shows that for $|(2 m+1) a|$ $\geq 1,\left\|\phi_{j} * \mu\right\|_{L^{\infty}} \leq C|(2 m+1) a|^{-1 / 2}$. These imply that the $L^{\infty}$ norm of $\widehat{R}$ is bounded by $C|\bar{a}|^{1 / 2}|(m+1)|^{-1 / 2}$. If $|(2 m+1) a| \leq 1$ then $\left\|\phi_{j} * \mu\right\|_{L^{\infty}} \leq C$ and consequently $\|\widehat{R}\|_{L^{\infty}} \leq C|a| \leq C|(m+1)|^{-1 / 2}|a|^{1 / 2}$. This proves the lemma.

2. Main theorem. For a fixed $\phi \in C_{\mathrm{c}}^{\infty}\left(\mathbb{H}^{n}\right)$ we define the local maximal function of the group $V_{t}$ by

$$
M f(\mathbf{z}, u)=\phi(\mathbf{z}, u) \sup _{0 \leq t \leq 1}\left|V_{t} f(\mathbf{z}, u)\right| .
$$

We have 
Theorem 1. Let $s>1 / 2$ and $f \in W^{s}$. Then

$$
\|M f\|_{L^{2}} \leq C\|f\|_{W^{s}} .
$$

Proof. Let $f \in L^{2}\left(\mathbb{H}^{n}\right)$. To estimate $\|M f\|_{L^{2}\left(\mathbb{H}^{n}\right)}$ we introduce a family of projections $P_{\alpha}$. Then we write

$$
\|M f\|_{L^{2}\left(\mathbb{H}^{n}\right)} \leq \sum_{\alpha}\left\|M P_{\alpha} f\right\|_{L^{2}\left(\mathbb{H}^{n}\right)}
$$

and we estimate each $\left\|M P_{\alpha} f\right\|_{L^{2}\left(\mathbb{H}^{n}\right)}$ separately.

We will use the abbreviation

$$
s \approx 2^{k} \quad \text { iff } \quad 2^{k} \leq s<2^{k+1} .
$$

For $k, l \in \mathbb{N}$ let

$$
\begin{aligned}
P_{k, l} f(\mathbf{z}, u) & =\sum_{\left\{\mathbf{m}:|\mathbf{m}| \approx 2^{k}\right\}} \int_{\left\{|a| \approx 2^{l}\right\}} e^{i u a} Q_{\mathbf{m}, a} f^{a}(\mathbf{z}) d a, \\
P_{0} f(\mathbf{z}, u) & =\sum_{\mathbf{m}} \int_{\{|a| \leq 1\}} e^{i u a} Q_{\mathbf{m}, a} f^{a}(\mathbf{z}) d a .
\end{aligned}
$$

Then obviously

$$
P_{0}+\sum_{k, l} P_{k, l}=\mathrm{Id}
$$

The maximal function of the theorem splits into the maximal functions

$$
\begin{aligned}
S_{k, l} f(\mathbf{z}, u) & =\sup _{0 \leq t \leq 1}\left|\psi(\mathbf{z}) \phi(u) P_{k, l} V_{t} f(\mathbf{z}, u)\right|, \\
S_{0} f(\mathbf{z}, u) & =\sup _{0 \leq t \leq 1}\left|\psi(\mathbf{z}) P_{0} V_{t} f(\mathbf{z}, u)\right|,
\end{aligned}
$$

where $\psi \in C_{\mathrm{c}}^{\infty}\left(\mathbb{C}^{n}\right), \widehat{\phi} \in C_{\mathrm{c}}^{\infty}(\mathbb{R}), \operatorname{supp} \widehat{\phi} \subset B(1)$.

We are going to estimate the norms $\left\|S_{k, l}\right\|_{W^{1 / 2+\varepsilon} \rightarrow L^{2}}$ and $\left\|S_{0}\right\|_{W^{1 / 2+\varepsilon} \rightarrow L^{2}}$. Then we sum up the estimates. With no loss of generality we may consider only the $\mathbf{m}$ 's in $I_{1}=\left\{\mathbf{m}: m_{1}=\max \left(m_{1}, \ldots, m_{n}\right)\right\}$.

Let $A=\left\{(\mathbf{m}, \mathbf{r}): m_{2}=r_{2}, \ldots, m_{n}=r_{n}, \mathbf{m}, \mathbf{r} \in I_{1}\right\}$. We fix $a$ and we note that $|\mathbf{m}|=|\mathbf{r}|$ and $(\mathbf{m}, \mathbf{r}) \in A$ imply $\mathbf{m}=\mathbf{r}$. By the orthogonality relations (0.1) for $P_{m, a}$ we have

$$
\begin{aligned}
& \int Q_{\mathbf{m}, a} f(\mathbf{z}) \overline{Q_{\mathbf{r}, a} f(\mathbf{z})} d z_{2} \ldots d z_{n} \\
& \quad=\int P_{m_{1}, a} P_{m_{2}, a} \ldots P_{m_{n}, a} f(\mathbf{z}) \overline{P_{r_{1}, a} P_{r_{2}, a} \ldots P_{r_{n}, a} f(\mathbf{z})} d z_{2} \ldots d z_{n}=0
\end{aligned}
$$

if $\left(m_{2}, \ldots, m_{n}\right) \neq\left(r_{2}, \ldots, r_{n}\right)$. In the formula above $P_{m_{i}, a}$ acts on the variable $z_{i}$. 
We begin by estimating the norm of $S_{0}$, making use of the Sobolev lemma. We have

$\left|S_{0} f(\mathbf{z}, u)\right|^{2} \leq C\left(\int_{\mathbb{R}}\left|\partial_{t}^{1 / 2+\varepsilon} V_{t} P_{0} f(\mathbf{z}, u)\right|^{2} \gamma(t) d t+\int_{\mathbb{R}}\left|V_{t} P_{0} f(\mathbf{z}, u)\right|^{2} \gamma(t) d t\right) \psi(\mathbf{z})$.

In what follows we assume that $\widehat{\gamma}$ is supported in the interval $[-1,1]$. Integrating with respect to $d \mathbf{z} d u$, by the Plancherel theorem applied to the Fourier transform in the central variable, we have

$$
\begin{aligned}
\int\left|S_{0} f(\mathbf{z}, u)\right|^{2} d \mathbf{z} d u \leq \int\left|\partial_{t}^{1 / 2+\varepsilon} P_{0} V_{t} f(\mathbf{z}, u)\right|^{2} \psi(\mathbf{z}) \gamma(t) d \mathbf{z} d u d t+C\|f\|_{L^{2}}^{2} \\
=C \iiint\left|\sum_{m} I_{\{0 \leq|a| \leq 1\}}(a) Q_{\mathbf{m}, a} f^{a}(\mathbf{z}) \partial_{t}^{1 / 2+\varepsilon} e^{i \lambda|\mathbf{m}|}(a) t\right|^{2} d a \gamma(t) d t \psi(\mathbf{z}) d \mathbf{z} \\
\quad+C\|f\|_{L^{2}}^{2} \\
\leq C \iiint \mid \sum_{\mathbf{m}} I_{\{C /|\mathbf{m}| \leq|a| \leq 1\}}(a)\left(\lambda_{\mathbf{m}}(a)\right)^{1 / 2+\varepsilon} \\
\quad \times\left. Q_{\mathbf{m}, a} f^{a}(\mathbf{z}) e^{i \lambda_{|\mathbf{m}|}(a) t}\right|^{2} d a \gamma(t) d t \psi(\mathbf{z}) d \mathbf{z}+\|f\|_{L^{2}}^{2} .
\end{aligned}
$$

In the last inequality we have used the fact that for $|a| \leq C|\mathbf{m}|^{-1}$, we have $\lambda_{|\mathbf{m}|}(a) \leq C$.

In the above sum the multiindices $\mathbf{m}$ belong to $I_{1}$. We enlarge the last expression by replacing the $\psi(\mathbf{z})$ by $\psi\left(z_{1}\right), \psi \in C_{\mathrm{c}}^{\infty}(\mathbb{C})$. Thus

$$
\begin{gathered}
\iiint\left|\sum_{\mathbf{m}} I_{\{C /|\mathbf{m}| \leq|a| \leq 1\}}(a)\left(\lambda_{|\mathbf{m}|}(a)\right)^{1 / 2+\varepsilon} Q_{\mathbf{m}, a} f^{a}(\mathbf{z}) e^{i \lambda_{|\mathbf{m}|}(a) t}\right|^{2} d a \gamma(t) d t \psi(\mathbf{z}) d \mathbf{z} \\
=\iint \sum_{\mathbf{m} \in I_{1}} \sum_{\mathbf{r} \in I_{1}} I_{\{C /|\mathbf{m}| \leq|a| \leq 1\}}(a) I_{\{C /|\mathbf{r}| \leq|a| \leq 1\}}(a)\left(\lambda_{|\mathbf{m}|}(a) \lambda_{|\mathbf{r}|}(a)\right)^{1 / 2+\varepsilon} \\
\times Q_{\mathbf{m}, a} f^{a}(\mathbf{z}) \overline{Q_{\mathbf{r}, a} f^{a}(\mathbf{z})} \widehat{\gamma}\left(\lambda_{|\mathbf{m}|}(a)-\lambda_{|\mathbf{r}|}(a)\right) d a \psi\left(z_{1}\right) d \mathbf{z} .
\end{gathered}
$$

By orthogonality of $P_{m, a}$ the last expression is equal to

$$
\begin{aligned}
& \int \sum_{(\mathbf{m}, \mathbf{r}) \in A} I_{\left\{C \max \left\{|\mathbf{m}|^{-1},|\mathbf{r}|^{-1}\right\} \leq|a| \leq 1\right\}}(a)\left(\lambda_{|\mathbf{m}|}(a) \lambda_{|\mathbf{r}|}(a)\right)^{1 / 2+\varepsilon} \\
& \times \int Q_{\mathbf{m}, a} f^{a}(\mathbf{z}) \overline{Q_{\mathbf{r}, a} f^{a}(\mathbf{z})} \psi\left(z_{1}\right) d \mathbf{z} \widehat{\gamma}\left(\lambda_{|\mathbf{m}|}(a)-\lambda_{|\mathbf{r}|}(a)\right) d a \\
& =\int \sum_{(\mathbf{m}, \mathbf{r}) \in A} I_{\left\{C \max \left\{|\mathbf{m}|^{-1},|\mathbf{r}|^{-1}\right\} \leq|a| \leq 1\right\}}(a)\left(\lambda_{|\mathbf{m}|}(a) \lambda_{|\mathbf{r}|}(a)\right)^{1 / 2+\varepsilon} \\
& \times \int Q_{\mathbf{m}, a} f^{a}(\mathbf{z}) \overline{Q_{\mathbf{r}, a} f^{a}(\mathbf{z})} \psi\left(z_{1}\right) d \mathbf{z} \widehat{\gamma}\left(2 m_{1}|a|-2 r_{1}|a|\right) d a
\end{aligned}
$$




$$
\begin{aligned}
\leq & \int \sum_{(\mathbf{m}, \mathbf{r}) \in A} I_{\left\{C \max \left\{|\mathbf{m}|^{-1},|\mathbf{r}|^{-1}\right\} \leq|a| \leq 1\right\}}(a)\left(\lambda_{|\mathbf{m}|}(a) \lambda_{|\mathbf{r}|}(a)\right)^{1 / 2+\varepsilon} \\
& \times\left(\frac{|a|}{|\mathbf{m}|+1} \frac{|a|}{|\mathbf{r}|+1}\right)^{1 / 4} \\
& \times\left\|Q_{\mathbf{m}, a} f^{a}\right\|\left\|Q_{\mathbf{r}, a} f^{a}\right\| \widehat{\gamma}\left(2\left(m_{1}-r_{1}\right)|a|\right) d a .
\end{aligned}
$$

To verify the last inequality we use Lemma 3 . The last expression is bounded by

$$
\begin{aligned}
S= & C \int \sum_{(\mathbf{m}, \mathbf{r}) \in A} I_{\left\{C \max \left\{|\mathbf{m}|^{-1},|\mathbf{r}|^{-1}\right\} \leq|a| \leq 1\right\}}(a)\left(\lambda_{|\mathbf{m}|}(a) \lambda_{|\mathbf{r}|}(a)\right)^{1 / 2+\varepsilon} \\
& \times\left(\left(\frac{|a|}{|\mathbf{m}|+1}\right)^{1 / 2}\left\|Q_{\mathbf{m}, a} f^{a}\right\|^{2}+\left(\frac{|a|}{|\mathbf{r}|+1}\right)^{1 / 2}\left\|Q_{\mathbf{r}, a} f^{a}\right\|^{2}\right) \widehat{\gamma}\left(2\left(m_{1}-r_{1}\right)|a|\right) d a .
\end{aligned}
$$

For fixed $\mathbf{r}$ we have

$$
\begin{aligned}
\sum_{\{\mathbf{m}:(\mathbf{m}, \mathbf{r}) \in A\}} I_{\{C} & \left.\max \left\{|\mathbf{m}|^{-1},|\mathbf{r}|^{-1}\right\} \leq|a| \leq 1\right\} \\
& \times\left(\frac{|a|}{|\mathbf{r}|+1}\right)^{1 / 2} \widehat{\gamma}\left(\left(m_{1}-r_{1}\right)|a|\right) \leq C\left(\lambda_{|\mathbf{m}|}(a)\right)^{1 / 2+2 \varepsilon} .
\end{aligned}
$$

In order to verify (2.3) we observe that for $\mathbf{m}, \mathbf{r}$, and $a$ as in (2.3) one can write

$$
\begin{gathered}
c \lambda_{|\mathbf{m}|}(a) \leq(|\mathbf{m}|+1)|a| \leq C \lambda_{|\mathbf{m}|}(a), \quad c \lambda_{|\mathbf{r}|}(a) \leq(|\mathbf{r}|+1)|a| \leq C \lambda_{|\mathbf{r}|}(a), \\
c|\mathbf{m}| \leq|\mathbf{r}| \leq C|\mathbf{m}| .
\end{gathered}
$$

To show the last inequality we observe that the conditions $\widehat{\gamma}\left(\left(m_{1}-r_{1}\right)|a|\right)$ $\neq 0,(\mathbf{m}, \mathbf{r}) \in A$ and $C \max \left\{|\mathbf{m}|^{-1},|\mathbf{r}|^{-1}\right\} \leq|a| \leq 1$ imply that || $\mathbf{m}|-| \mathbf{r}||$ $\leq C \min \{|\mathbf{r}|,|\mathbf{m}|\}$. Also

$$
\sharp\left\{\mathbf{m}:(\mathbf{m}, \mathbf{r}) \in A,\left|r_{1}-m_{1}\right||a| \in \operatorname{supp} \widehat{\gamma}\right\} \leq C /|a| .
$$

Now (2.3) follows by an easy calculation.

By (2.3), $S$ is dominated by

$$
2 \int \sum_{\mathbf{r}} I_{\left\{C \mathbf{r}^{-1} \leq|a| \leq 1\right\}}(a)\left(\lambda_{|\mathbf{r}|}(a)\right)^{1 / 2+\varepsilon}\left\|Q_{\mathbf{r}, a} f^{a}\right\|^{2} d a \leq\|f\|_{W^{1 / 2+\varepsilon}}^{2} .
$$

We are going to estimate $\left\|S_{k, l} f(\mathbf{z}, u)\right\|_{L^{2}}$ in a similar way. Without loss of generality, we can consider only

$$
S_{k, l}^{1} f(\mathbf{z}, u)=\sup _{0 \leq t \leq 1}\left|\psi\left(z_{1}\right) \phi(u) P_{k, l}^{1} V_{t} f(\mathbf{z}, u)\right|
$$

where

$$
P_{k, l}^{1} f(\mathbf{z}, u)=\sum_{\left\{\mathbf{m} \in I_{1}:|\mathbf{m}| \approx 2^{k}\right\}} \int_{\left\{|a| \approx 2^{l}\right\}} e^{i u a} Q_{\mathbf{m}, a} f^{a}(\mathbf{z}) d a .
$$


Again by the Sobolev lemma, the norm $\left\|S_{k, l}^{1} f(\mathbf{z}, u)\right\|_{L^{2}}^{2}$ is controlled by

$$
\begin{aligned}
& \iiint\left|\sum_{\left\{\mathbf{m} \in I_{1}:|\mathbf{m}| \approx 2^{k}\right\}} \int_{\left\{|a| \approx 2^{l}\right\}} e^{i u a+i t \lambda_{|\mathbf{m}|}(a)} \lambda_{|\mathbf{m}|}^{1 / 2+\varepsilon}(a) Q_{\mathbf{m}, a} f^{a}(\mathbf{z}) d a\right|^{2} \\
& \times \psi\left(z_{1}\right) \phi(u) d \mathbf{z} d u \gamma(t) d t \\
& =\iiint_{\left\{\mathbf{m}, \mathbf{r}:|\mathbf{m}|,|\mathbf{r}| \approx 2^{k}, \mathbf{r} \in I_{1}\right\}} \iint e^{i u\left(a_{1}-a_{2}\right)+i t\left(\lambda_{|\mathbf{m}|}\left(a_{1}\right)-\lambda_{|\mathbf{r}|}\left(a_{2}\right)\right)} \\
& \times I_{\left\{|a| \approx 2^{l}\right\}}\left(a_{1}\right) I_{\left\{|a| \approx 2^{l}\right\}}\left(a_{2}\right)\left(\lambda_{|\mathbf{m}|}\left(a_{1}\right) \lambda_{|\mathbf{r}|}\left(a_{2}\right)\right)^{1 / 2+\varepsilon} \\
& \times Q_{\mathbf{m}, a_{1}} f^{a_{1}}(\mathbf{z}) Q_{\mathbf{r}, a_{2}} f^{a_{2}}(\mathbf{z}) \psi\left(z_{1}\right) d \mathbf{z} \phi(u) d u \gamma(t) d t d a_{1} d a_{2} \\
& =\sum_{\left\{\mathbf{m}, \mathbf{r}:|\mathbf{r}| \approx 2^{k}, \mathbf{m}, \mathbf{r} \in I_{1}\right\}} \iint I_{\left\{|a| \approx 2^{l}\right\}}\left(a_{1}\right) I_{\left\{|a| \approx 2^{l}\right\}}\left(a_{2}\right)\left(\lambda_{|\mathbf{m}|}\left(a_{1}\right) \lambda_{|\mathbf{r}|}\left(a_{2}\right)\right)^{1 / 2+\varepsilon} \\
& \times Q_{\mathbf{m}, a_{1}} f^{a_{1}}(\mathbf{z}) Q_{\mathbf{r}, a_{2}} f^{a_{2}}(\mathbf{z}) \psi\left(z_{1}\right) d \mathbf{z} \\
& \times \widehat{\phi}\left(a_{1}-a_{2}\right) \widehat{\gamma}\left(\lambda_{|\mathbf{m}|}\left(a_{1}\right)-\lambda_{|\mathbf{r}|}\left(a_{2}\right)\right) d a_{1} d a_{2} \\
& =\sum_{\left\{(\mathbf{m}, \mathbf{r}) \in A: \mathbf{m}, \mathbf{r} \in I_{1},|\mathbf{m}|,|\mathbf{r}| \approx 2^{k}\right\}} \iint I_{\left\{|a| \approx 2^{l}\right\}}\left(a_{1}\right) I_{\left\{|a| \approx 2^{l}\right\}}\left(a_{2}\right) \\
& \times\left(\lambda_{|\mathbf{m}|}\left(a_{1}\right) \lambda_{|\mathbf{r}|}\left(a_{2}\right)\right)^{1 / 2+\varepsilon} \int Q_{\mathbf{m}, a} f^{a}(\mathbf{z}) Q_{\mathbf{r}, a} f^{a}(\mathbf{z}) \psi\left(z_{1}\right) d \mathbf{z} \\
& \times \widehat{\phi}\left(a_{1}-a_{2}\right) \widehat{\gamma}\left(\lambda_{|\mathbf{m}|}\left(a_{1}\right)-\lambda_{|\mathbf{r}|}\left(a_{2}\right)\right) d a_{1} d a_{2} \\
& \leq 2 \int \sum_{\left\{\mathbf{r} \in I_{1}:|\mathbf{r}| \approx 2^{k}\right\}} \int_{\left\{\mathbf{m}:(\mathbf{m}, \mathbf{r}) \in A,|\mathbf{m}| \approx 2^{k}\right\}} I_{\left\{|a| \approx 2^{l}\right\}}\left(a_{1}\right) I_{\left\{|a| \approx 2^{l}\right\}}\left(a_{2}\right) \\
& \times\left(\lambda_{|\mathbf{m}|}\left(a_{1}\right) \lambda_{|\mathbf{r}|}\left(a_{2}\right)\right)^{1 / 2+\varepsilon} \widehat{\gamma}\left(\lambda_{|\mathbf{m}|}\left(a_{1}\right)-\lambda_{|\mathbf{r}|}\left(a_{2}\right)\right) \widehat{\phi}\left(a_{1}-a_{2}\right) d a_{1} \\
& \times \int\left|Q_{\mathbf{r}, a_{2}} f^{a_{2}}(\mathbf{z})\right|^{2} \psi\left(z_{1}\right) d \mathbf{z} d a_{2}=J .
\end{aligned}
$$

For fixed $\mathbf{r}$ and $a_{2}$ we have

$$
\begin{aligned}
& \int_{\left\{\mathbf{m}:(\mathbf{m}, \mathbf{r}) \in A,|\mathbf{m}| \approx 2^{k}\right\}} I_{\left\{|a| \approx 2^{l}\right\}}\left(a_{1}\right) I_{\left\{|a| \approx 2^{l}\right\}}\left(a_{2}\right) \\
& \quad \times\left(\lambda_{|\mathbf{m}|}\left(a_{1}\right) \lambda_{|\mathbf{r}|}\left(a_{2}\right)\right)^{1 / 2+\varepsilon} \widehat{\phi}\left(a_{1}-a_{2}\right) \widehat{\gamma}\left(\lambda_{|\mathbf{m}|}\left(a_{1}\right)-\lambda_{|\mathbf{r}|}\left(a_{2}\right)\right) d a_{1} \\
& \leq\left(2^{k}+2^{l}\right)^{(1+2 \varepsilon)} 2^{2 l \varepsilon} .
\end{aligned}
$$

To see (2.4) we observe that $\frac{d}{d a_{1}} \lambda_{|\mathbf{m}|}\left(a_{1}\right)=\left((2|\mathbf{m}|+n)+2\left|a_{1}\right|\right) \operatorname{sgn}\left(a_{1}\right)$. So the measure of $\left\{a_{1}: \lambda_{|\mathbf{m}|}\left(a_{1}\right)-\lambda_{|\mathbf{r}|}\left(a_{2}\right) \in \operatorname{supp} \hat{\gamma}\right\}$ is dominated by $C /\left(2^{k}+2^{l}\right)$. Hence

$$
\int \widehat{\gamma}\left(\lambda_{|\mathbf{m}|}\left(a_{1}\right)-\lambda_{|\mathbf{r}|}\left(a_{2}\right)\right) d a_{1} \leq \frac{C}{2^{k}+2^{l}} .
$$


Also

$$
\begin{aligned}
& \sharp\left\{\mathbf{m} \approx 2^{k}:(\mathbf{m}, \mathbf{r}) \in A \text { and } \exists_{a_{1} \approx 2^{l}} \lambda_{|\mathbf{m}|}\left(a_{1}\right)-\lambda_{|\mathbf{r}|}\left(a_{2}\right) \in \operatorname{supp} \widehat{\gamma}\right. \\
& \text { and } \left.a_{1}-a_{2} \in \operatorname{supp} \widehat{\phi}\right\} \leq C \max \left\{1,|\mathbf{r}| / 2^{l}\right\} .
\end{aligned}
$$

Combining (2.5) and (2.6) gives (2.4).

Hence by Lemma 2 and (2.4) we obtain the desired estimate for $J$ :

$$
\begin{aligned}
J & \leq \int \sum_{\left\{\mathbf{r} \in I_{1}:|\mathbf{r}| \approx 2^{k}\right\}} 2^{2 l \varepsilon}\left(\frac{2^{l}}{2^{k}+2^{l}}\right)^{1 / 2}\left(2^{k}+2^{l}\right)^{1+2 \varepsilon}\left\|Q_{\mathbf{r}, a} f^{a}\right\|_{L^{2}}^{2} d a \\
& \leq \int \sum_{\left\{\mathbf{r} \in I_{1}:|\mathbf{r}| \approx 2^{k}\right\}}\left(2^{k} 2^{l}+2^{2 l}\right)^{1 / 2+2 \varepsilon}\left\|Q_{\mathbf{r}, a} f^{a}\right\|_{L^{2}}^{2} d a \leq C\|f\|_{W^{1 / 2+8 \varepsilon}} 2^{-(k+l) \varepsilon}
\end{aligned}
$$

Summing up the estimates for $S_{0}$ and $S_{k, l}$ we get the theorem.

REMARK. The above theorem combined with the estimates obtained in [Z] allows one to state a slightly sharper result. This requires a different definition of the scale of Sobolev spaces. We do not go into details here.

\section{References}

[B] J. Bourgain, A remark on Schrödinger operators, Israel J. Math. 77 (1992), $1-16$.

[Car] A. Carbery, Radial Fourier multipliers and associated maximal functions, in: Recent Progress in Fourier Analysis, North-Holland Math. Stud. 111, NorthHolland, 1985, 49-56.

[C] L. Carleson, Some analytical problems related to statistical mechanics, in: Euclidean Harmonic Analysis, Lecture Notes in Math. 779, Springer, 1979, 5-45.

[Cw] M. Cowling, Pointwise behavior of solutions to Schrödinger equations, in: Harmonic Analysis, Lecture Notes in Math. 992, Springer, 1983, 83-90.

[DK] B. E. J. Dahlberg and C. E. Kenig, A note on the almost everywhere behavior of solutions to the Schrödinger equation, in: Harmonic Analysis, Lecture Notes in Math. 908, Springer, 1982, 205-209.

[E1] A. Erdélyi, Asymptotic forms for Laguerre polynomials, J. Indian Math. Soc. 24 (1960), 235-250.

[E2] A. Erdélyi, W. Magnus, F. Oberhettinger and G. F. Tricomi, Higher Transcendental Functions, McGraw-Hill, New York, 1953.

[HR] A. Hulanicki and F. Ricci, A Tauberian theorem and tangential convergence of bounded harmonic functions on balls in $\mathbb{C}^{n}$, Invent. Math. 62 (1980), 325-331.

[KPV1] C. E. Kenig, G. Ponce and L. Vega, Oscillatory integrals and regularity of dispersive equations, Indiana Univ. Math. J. 40 (1991), 33-69.

[KPV2] -, 一, 一, Well-posedness of the initial value problem for the Korteweg-de Vries equation, J. Amer. Math. Soc. 4 (1991), 323-347.

[KR] C. E. Kenig and A. Ruiz, A strong type $(2,2)$ estimate for a maximal operator associated to the Schrödinger equation, Trans. Amer. Math. Soc. 280 (1983), 239-246. 
[Mo] A. Moyua, A. Vargas and L. Vega, Schrödinger maximal function and restriction properties of the Fourier transform, Internat. Math. Res. Notices 1996, no. 16, 793-815.

[M] D. Müller, A restriction theorem for the Heisenberg group, Ann. of Math. 131 (1990), 567-587.

[MR] D. Müller and F. Ricci, Analysis of second order differential operators on Heisenberg groups. I, Invent. Math. 101 (1990), 545-582.

[NS] E. Nelson and W. F. Stinespring, Representations of elliptic operators in an enveloping algebra, Amer. J. Math. 81 (1959), 547-560.

[P] E. Prestini, Radial functions and regularity of solutions to the Schrödinger equation, Monatsh. Math. 109 (1990), 135-143.

[SS] P. Sjögren and P. Sjölin, Convergence properties for the time dependent Schrödinger equation, Ann. Acad. Sci. Fenn. Ser. A.I. Math. 14 (1989), 13-25.

[S1] P. Sjölin, Regularity of solutions to the Schrödinger equations, Duke J. Math. 55 (1987), 699-715.

[S2] - Radial functions and maximal estimates for solutions to the Schrödinger equation, J. Austral. Math. Soc. Ser. A 59 (1995), 134-142.

[S3] - Global maximal estimates for solutions to the Schrödinger equation, Studia Math. 110 (1994), 105-114.

[Sz] G. Szegö, Orthogonal Polynomials, Colloq. Publ. 23, Amer. Math. Soc., 1939.

[V] L. Vega, Schrödinger equations: pointwise convergence to the initial data, Proc. Amer. Math. Soc. 102 (1988), 874-878.

[Z] J. Zienkiewicz, Initial value problem for the time dependent Schrödinger equation on the Heisenberg group, Studia Math. 122 (1997), 15-37.

Institute of Mathematics

University of Wrocław

Pl. Grunwaldzki 2/4

50-384 Wrocław, Poland

E-mail: zenek@math.uni.wroc.pl

Received November 21, 1997

Revised version November 28, 2002 